\title{
Editorial
}

Rechtsmedizin 2016 -26:1

DOI 10.1007/s00194-015-0074-x

๑) Springer-Verlag Berlin Heidelberg 2016

CrossMark

Nach einem Vierteljahrhundert und seither drei Herausgebern (Michael Staak 1991-1999, Gunther Geserick 1999-2002 und Klaus Püschel seit 2003) soll die geschäftsführende Herausgeberschaft nunmehr vorübergehend von zwei Personen wahrgenommen werden. An die Seite von Klaus Püschel wird der bisherige Herausgeber der Rubrik Rechtsreport, Reinhard Dettmeyer, treten.

Die beiden werden gemeinsam die Förderung der Fortentwicklung der Zeitschrift Rechtsmedizin betreiben. Die Konzeption der Zeitschrift, wie sie sich bis zum heutigen Tage erfolgreich entwickelt hat, soll beibehalten werden, gelang doch damit in der Vergangenheit die Aufnahme unserer Fachzeitschrift in die Journal Citation Reports (JCR) nach Annahme durch das Institute for Scientific Information (I.S.I.) in Philadelphia. Mittlerweile verfügt die Rechtsmedizin über einen ImpactFaktor; Publikationen in der Rechtsmedizin werden bei Habilitationen und Berufungen berücksichtigt. Heute bieten sämtliche Universitätsbibliotheken im deutschsprachigen Raum einen Onlinezugang an, sodass sich die Akzeptanz der Rechtsmedizin und ihr gestiegenes Renommee zusätzlich in steigenden Onlinenutzerzahlen zeigt. Diese erfreuliche Entwicklung soll auch in den nächsten Jahren fortgesetzt werden, gemeinsam und kollegial mit den Rubrikherausgebern, den Mitgliedern des Editorial Board und des Scientific Advisory Board.

Unterstützt durch die Deutsche Gesellschaft für Rechtsmedizin (DGRM) soll in unserer Zeitschrift das gesamte Spektrum des Fachs thematisch repräsentiert sein, mit im Einzelfall auch englischsprachigen Beiträgen, mit großem Verständnis gerade für interdisziplinäre Kooperationen und für den wissenschaftlichen Austausch auf

\author{
K. Püschel ${ }^{1} \cdot$ R. Dettmeyer ${ }^{2}$ \\ ${ }^{1}$ Institut für Rechtsmedizin, Universitätsklinikum Hamburg-Eppendorf, Hamburg, Deutschland \\ ${ }^{2}$ Institut für Rechtsmedizin, Justus-Liebig-Universität, Universitätsklinikum \\ Gießen und Marburg GmbH, Gießen, Deutschland
}

\section{Gemeinsame Herausgeberschaft}

nationaler sowie internationaler Ebene. Dies wird ebenfalls weiterhin garantiert durch die kompetente Betreuung seitens des Springer-Verlags, allen voran durch den dortigen, dankenswerten, stets zuverlässigen Einsatz von Frau Lorenz und durch das fortdauernde Bemühen um die Förderung der Qualität eingereichter Beiträge.

\section{》) Die Rechtsmedizin \\ soll auch weiterhin unser \\ "wissenschaftliches \\ Sprachrohr" bleiben}

Die zukünftig gemeinsame Herausgeberschaft soll darüber hinaus zum Anlass genommen werden, allen Reviewern und Reviewerinnen ganz herzlich für ihre seit Jahren uneigennützige, prompte und fristgerechte Unterstützung bei der Begutachtung von Manuskripten zu danken. Ohne diese Bereitschaft zur Begutachtung wäre das heutige hohe Qualitätsniveau nicht erreicht worden. Verbunden werden darf dieser Dank mit der Bitte, auch zukünftig im Einzelfall zur Verfügung zu stehen.

Im Editorial zum letzten Herausgeberwechsel im Jahr 2003 wurde die Rechtsmedizin als unser „wissenschaftliches Sprachrohr“ bezeichnet. Dies soll so bleiben, zur Förderung der historisch und aktuell wichtigen Reputation unserer Fachgesellschaft, der Deutschen Gesellschaft für Rechtsmedizin (DGRM), auf nationaler und internationaler Ebene.

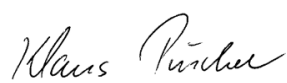

Prof. Dr. Klaus Püschel<smiles>CC1C=CC(C=CC2CC2)C=C1</smiles>

Prof. Dr. Dr. Reinhard Dettmeyer

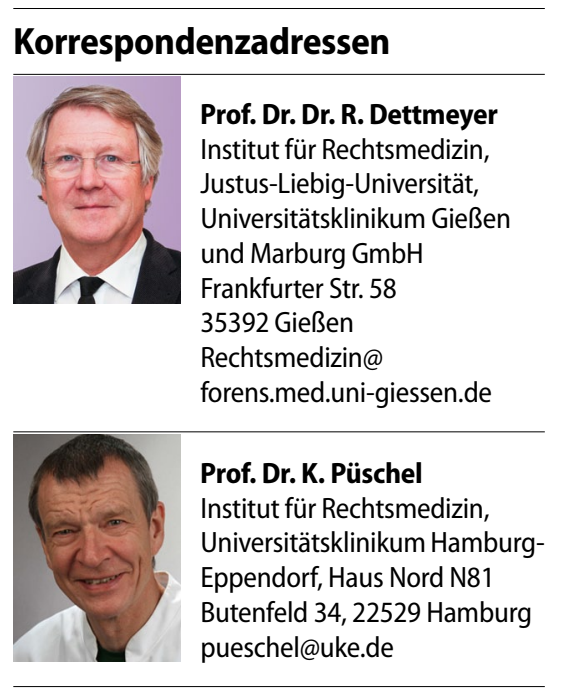

